\title{
An MHD Model of Be Stars with Disks
}

\author{
A. E. Dudorov
}

Chelyabinsk State University, 124 Kashirinych St., Chelyabinsk, 454136 Russia

R. E. Pudritz

McMaster University, Hamilton, Ontario, L8S 4M1 Canada

\begin{abstract}
An investigation of the formation and evolution of Be stars show that these stars could have dipolar fossil magnetic fields. This magnetic field should constrain the circumstellar magnetic disk and influence its dynamical features. For studying these effects we refine the alphamodel of accretion disks of Shakura and Sunyaev by incorporating into this model the evolution of large-scale magnetic fields. In the frame of our model we can investigate the rotational history of the star with the disk and the ionization and thermal properties of disks. We show in particular that in the magnetopause region a current sheet can form that can generate X-ray radiation.
\end{abstract}

\section{Introduction}

Be stars are excellent study objects for the theory of fossil magnetic fields (see Dudorov 1990, 1995, 1998). The magnetic flux of stars in the frame of this theory is considered as a fossil (residual or relic) part of the magnetic flux of the parent protostellar clouds. Let us estimate as a proof the basic timescales.

The nuclear timescale of main sequence stars is:

$$
t_{N} \approx 10^{10}(M / L), L \simeq M^{4}, t_{N} \approx 10^{10} M^{-3} .
$$

For a Be star with mass $M=10 \mathrm{M}_{\odot}, t_{N} \approx 10^{7} \mathrm{yrs}$. The timescale for Ohmic dissipation of a magnetic field is:

$$
t_{\mathrm{Ohm}}=\frac{R^{2}}{\nu_{m}} \approx 10^{10}\left(\frac{R}{\mathrm{R}_{\odot}}\right)^{2}
$$

where $\nu_{m}$ is the coefficient of magnetic diffusivity. Taking into account that $R \simeq M^{q_{r}}$ with $q_{r} \approx 0.6$ for the upper part of the main sequence we estimate

$$
t_{\mathrm{Ohm}} \approx 10^{10} \mathrm{M}_{\odot}^{1.2} \approx 10^{11} \mathrm{yrs}
$$

for such a Be star. These estimates allow us to draw the following conclusions:

1. Be stars are young stars from the point of view of star formation theory. Their $t_{n} \approx t_{S F}$ are approximately $\approx 10^{7} \mathrm{yrs}$. Therefore they take part of the contemporary star formation process (CSFP). 
2. CSFP happens in interstellar magnetized rotating clouds. There are many observational confirmations of this statement (Dudorov 1995).

3. In the convective cores of Be stars the $\alpha-\omega$ dynamo process may work. The diffusion timescale of a dynamo-generated magnetic field throughout the radiative interiors up to the envelopes of these stars is: $t_{\mathrm{diff}}=t_{\mathrm{Ohm}} \approx$ $10^{11} \mathrm{yrs} \gg t_{N} \approx 10^{7} \mathrm{yrs}$.

4. Items 1-3 show that Be stars should have fossil magnetic fields.

\section{Theory of fossil magnetic fields}

\subsection{One and half approximation}

The hypothesis of fossil (relic, primordial, ...) magnetic fields was formulated by Cowling (1945), Spitzer (1958) and Mestel (1967) more than 30 years ago. They stated the so-called Problem of Magnetic Flux. Spitzer (1958) argued that in the process of star formation there are stages when the electric conductivity must be equal to zero. Therefore the fossil magnetic field of stars cannot exceed the value of the interstellar field, $B \approx 10^{-4} \mathrm{G}$. Mestel (1967) estimated that the magnetic field of stars must exceed the virial value in the case of conservation of magnetic flux.

We now consider the theory of fossil magnetic fields (Dudorov, 1990). The main goal of this theory is to study the evolution of magnetic flux during the formation and evolution of stars. It is very important in the frame of this theory to investigate the efficiency of ambipolar and Ohmic diffusion and the interaction of the magnetic field with rotation and turbulence, which all can reduce the magnetic flux of protostellar clouds.

For these investigations we use the radiative MHD equations in "diffusional" variables, which consist of the usual radiative MHD and additional equations (see Dudorov, 1990):

$$
\begin{gathered}
\frac{\partial x}{\partial t}+(\vec{v} \cdot \nabla) x=\frac{S_{p}}{\rho}-\frac{\nabla\left(x \rho \vec{v}_{m}\right)}{\rho}, \\
\frac{\partial \vec{v}_{m}}{\partial t}+(\vec{v} \cdot \nabla) \vec{v}_{m}=\frac{c_{s}^{2} \frac{\nabla x}{x}}{\rho_{i}}+\frac{\vec{F}_{e m}}{x \rho}-\eta_{i n} \rho \vec{v}_{m}, \\
\frac{\partial \vec{B}}{\partial t}=\operatorname{curl}\left[\left(\vec{v}+\vec{v}_{m}\right) \times \vec{B}\right]-\nabla \times\left(\eta_{m} \nabla \times \vec{B}\right), \operatorname{div} \vec{B}=0,
\end{gathered}
$$

where $x=\rho_{p} /\left(\rho_{p}+\rho_{n}\right)$ is the ionization degree, $\vec{v}_{m}=\vec{v}-\vec{v}_{p}$ is the velocity of ambipolar diffusion, $\rho_{p}$ and $\rho_{n}$ are the densities of charge and neutral components, $\vec{v}_{p}$ is the velocity of the charge component, and $S_{p}$ is the source function for the charges. Other quantities are used in their usual astrophysical meaning.

Equations (4-6) allow us to investigate nonstationary and nonequilibrium ionization and nonstationary magnetic ambipolar diffusion (MAD). We elaborate the one and half $(1.5 \mathrm{D})$ approximation for the numerical solution of the radiative MHD equations in the diffusional variables. The numerical simulations are carried out with the help of a modified Lax-Vendroff scheme. 
The numerical calculations show that the magnetic field is frozen in the gas in the course of a transparent collapse, during which a quasi-radial geometry is acquired. If the ionization state is determined by cosmic rays and radioactive elements, MAD diminishes the magnetic field in the opaque protostellar core when the central density $n_{c} \approx\left[10^{5} \cdot n_{0}, 10^{9} \cdot n_{0}\right]$, where $n_{0}=10^{4}-10^{5} \mathrm{~cm}^{-3}$ is the initial density of protostellar clouds. The adiabatic heating of the opaque core switches on the thermal evaporation of grains and the thermal ionization of light elements like $\mathrm{K}, \mathrm{Na}, \mathrm{Al}$ etc. with low ionization potentials. Therefore in the regions with temperature $T \approx 4000-5000 \mathrm{~K}$ the magnetic field will be immersed into the whole gas again. The zone of powerful MAD moves to the surface in the course of the evolution of protostars and young stars coinciding with the region of minimal ionization degree.

The attenuation of the frozen-in magnetic flux is $\approx 10^{-2}$ for a star with 10 $\mathrm{M}_{\odot}$. The surface magnetic field (before the interaction with convection) will be

$$
B_{s} \approx B_{s 0} \cdot\left(M / \mathrm{M}_{\odot}\right)^{0.25-0.35}, B_{s 0} \approx F\left(\tau_{C R}, Z_{R E}, Z_{q},\right) .
$$

Taking common values for the "optical" depth of cosmic rays $\tau_{C R}$, the abundance of radioactive elements $Z_{R E}$ as well as for the radius and abundance of grains $a$ and $q$, we obtain $B_{s} \approx 10-300 G$ for normal stars and $B_{s 0} \leq 2000-3000 G$ for the magnetic CP stars. The quantity $B_{s 0}$ depends also on the initial magnetic field of the protostellar cloud. The strength of the magnetic field increases towards the center of the star and has in the core the value $\approx(0.1-10) \cdot 10^{6} \mathrm{G}$ depending on the stellar mass. Therefore magnetic ambipolar diffusion solves the Magnetic Flux Problem in the process of star formation (see Dudorov 1990).

Since convection is absent in the envelope of early-type stars, the magnetic field can suppress completely the weak convective instability in this case (Dudorov and Tutukov 1990). The Ohmic decay time is sufficiently larger than the evolutionary timescale for these stars. Convection in the cores of B stars entangles the fossil magnetic field and diminishes its strength up to the limit of nonlinear stabilization of dynamo mechanisms. The timescale for diffusion to the surface exceeds the evolutionary timescale for a dynamo generated magnetic field. Therefore a fossil magnetic field may be conserved in hot stars, and in $\mathrm{Be}$ star in particular.

\subsection{D MHD simulations}

We have created a new 2D numerical code to investigate various astrophysical MHD problems and proved the basic conclusions of the theory of fossil magnetic fields. Our code is based on the TVD-scheme for the solution of MHD problems (Dudorov et al. 1999a, b). This monotonic scheme has a high order of approximation in the smoothing regions of the solution.

On the base of this scheme, the code "Moon" which can simulate MHD flows both in the $1 \mathrm{D}$ and $2 \mathrm{D}$ case was developed. It has a module to solve the Poisson equation for the gravitational potential, permitting the computation of the gravitational collapse of protostellar clouds. The Poisson equation is solved by the Douglas-Rachford ADI method. The code was tested on many problems with known exact or approximate analytical solutions, demonstrating its capability for the solution of a wide class of MHD-problems. 
Numerical computations of the collapse of a magnetized protostellar cloud have confirmed earlier results obtained in the frame of $1.5 \mathrm{D}$ approximation. Magnetic fields lead to the flattening of collapsing clouds at late stages of the contraction. Numerical simulations show that for sufficiently large values of the initial magnetic field, the degree of flattening is proportional to $\varepsilon_{m}^{-1 / 2}$, where $\varepsilon_{m}$ is the initial ratio of the magnetic to the gravitational energy.

We also performed simulations of a collapse of a rotating magnetized cloud with the initial value $\varepsilon_{m}=0.2$ and an initial ratio of magnetic to kinetic rotational energy $\varepsilon_{m \omega}=5$. The results show that a flat fast-rotating disk is formed around an opaque core (protostars) within the free-fall time. The parameters of such a disk are quite similar to the parameters of accretion onto a protoplanetary disk as observed in the recently discovered system $\mathrm{HH} 4796 \mathrm{~A}$.

\subsection{Conclusions from the theory}

The theory of fossil magnetic field has a large number of applications (see Dudorov $1990,1995,1998$ ). In the frame of this theory a Be star can have a fossil magnetic field with a dipole (or quadrupolar) geometry. They also may have a primordial disk with a magnetic field directed parallel but opposite to the moment of the stellar field. The matter of the disk accrete onto the star along the magnetic equators. The stellar wind of Be stars streams mainly from the magnetic poles along the magnetic fieldlines, adding matter to the disk. In such a situation we may use the Shakura and Sunyaev (1973) diskmodel for the description of Be-star disks. For the clarification of the magnetic properties of the disks we should incorporate the magnetic field into this model.

\section{MHD model of Be-star disks}

\subsection{Large scale magnetic fields}

Shakura and Sunyaev (1972) constructed the well-known model of a geometrically thin and optically thick disk with a prescribed turbulent viscosity (the SS model). They claimed that the turbulent viscosity may be produced by a turbulent magnetic field. We will incorporate a large scale magnetic field into the SS model. We need to find the solution of the induction eq. (6). Let us consider the case in which the velocity of the MAD $\vec{v}_{m}=0$, the magnetic diffusivity $\eta_{m}$ is constant, and the magnetic and velocity fields in a cylindrical coordinate system $\{r, \varphi, z\}$ have the following particular orientation

$$
\vec{B}=\left\{0, B_{\varphi}(r, z), B_{z}(r)\right\}, \vec{v}=\left\{v_{r}(r), v_{\varphi}(r, z), 0\right\} .
$$

For a stationary disk the induction equation turns into:

$$
\begin{gathered}
B_{z} \frac{\partial v_{\varphi}}{\partial z}-\frac{\partial}{\partial r}\left(v_{r} B_{\varphi}\right)+\eta_{m}\left(\Delta B_{\varphi}-\frac{B_{\varphi}}{r^{2}}\right)=0 \\
-\frac{1}{r} \frac{\partial}{\partial r}\left(r v_{r} B_{z}\right)+\eta_{m} \Delta B_{z}=0
\end{gathered}
$$

Equations (9-10) can be solved in the frame of the SS approximations for a thin disk. Neglecting in eq. (9) the radial advection and in eq. (10) the magnetic 
diffusivity it is easy to find the following solutions:

$$
\begin{gathered}
B_{z}=B_{z 0} \frac{\Sigma}{\Sigma_{0}}, \\
B_{\varphi}=\frac{3}{2} B_{z 0}\left(\frac{\Sigma}{\Sigma_{0}}\right)\left(\Omega_{k} \frac{H^{2}}{\eta_{m}}\right)\left(\frac{H}{R}\right),
\end{gathered}
$$

where $B_{z 0}$ is the initial fossil magnetic field, $\Sigma$ is the surface density, $\Omega_{k}$ is the Keplerian velocity, and $H$ is the semithickness of the disk.

\subsection{Viscosity}

The coefficient of turbulent viscosity $\nu_{m}$ is determined in the SS model by the parameter $\alpha \approx(0.01-0.1)$ in the relation $\nu_{t}=\alpha c_{s} H$, where $c_{s}$ is the sound speed. The magnetic diffusivity modifies the turbulent viscosity. We can write this in the MHD model $\alpha=\alpha_{S S}+\alpha_{m}$. The magnetic $\alpha_{m}$ may be determined formally from the relation $\eta_{m}=\alpha_{m} v_{A} H$, where $v_{A}$ is the Alfvén velocity. In the common case

$$
\eta_{m}=\eta_{\mathrm{Ohm}}+\eta_{\mathrm{b}}+\eta_{\mathrm{in}}+\eta_{\mathrm{AD}}
$$

where $\eta_{i}, i=\mathrm{Ohm}, \mathrm{b}$, in, $\mathrm{AD}$ ) is the magnetic diffusivity caused by Ohmic diffusion, buoyancy, instabilities and ambipolar diffusion, respectively. For simplicity we take into account the Ohmic diffusion when

$$
\eta_{\mathrm{Ohm}}=480 x^{-1} T^{1 / 2},
$$

where $x$ is the ionization fraction.

\subsection{Analytical solution}

To close eqs. $(11-12,14)$ we need to add to this system the ionization eq. (4). In the case of stationary ionization the equilibrium between ionization by cosmic rays and recombination to grains, eq. (4) has the following analytical solution (see Dudorov 1990): $x=0.13 / n$.

For a Kramer's law of opacity it is easy to find in terms of the SS model the analytical solution of the system $(11-12,14)$ :

$$
\begin{gathered}
x=A_{x} \alpha^{7 / 10} M^{5 / 8}(\dot{M})^{11 / 20} R^{15 / 8} f^{11 / 20} \\
B_{z}=A_{B_{z}} \alpha^{-4 / 5} M^{1 / 4}(\dot{M})^{7 / 10} R^{-3 / 4} f^{7 / 10} \\
B_{\varphi}=A_{B_{\varphi}} \alpha^{-11 / 10} M^{-3 / 8}(\dot{M})^{23 / 20} R^{1 / 8} f^{23 / 20}
\end{gathered}
$$

where

$$
f=1-\left(\frac{R_{d}}{R}\right)^{1 / 2},
$$

where $R_{d}$ is the inner edge of the disk. To specify this solution we consider a $\mathrm{Be}$ star with mass $M=10 \mathrm{M}_{\odot}$ and radius $R_{*}=8 \mathrm{R}_{\odot}$. For the initial magnetic field of protostellar cloud we take $B_{0}=10-5 \mathrm{G}, \alpha=0.1$ and a typical accretion rate of Be stars $\dot{M}=10^{-8} \mathrm{M}_{\odot} / \mathrm{yr}$

$$
B_{z}=5.3 R^{-3 / 4} f^{7 / 10} \mathrm{G}, B_{\varphi}=7.8 R^{1 / 8} f^{23 / 20} \mathrm{G} .
$$


If the star has a dipole magnetic field with a magnetic induction on the surface of $B_{s}=200 \mathrm{G}$, then the inner radius of the disk is determined by the magnetospheric radius $R_{d}=R_{m} \approx 3 R_{*}$. Near to the inner edge of the disk the toroidal component of the magnetic field exceeds the poloidal component by a factor 1.5 . In this region $f \approx 1$. Therefore the poloidal field decreases inversely with the radius, whereas the toroidal field increases slightly with radius.

The radius of corotation for a star with rotational velocity $v_{\text {rot }} \approx 200 \mathrm{~km} / \mathrm{s}$ is $R_{\text {co }} \approx 9.8 R_{*}$. Therefore Be stars are accreting objects.

\section{Conclusion}

Our consideration shows that Be stars should have a fossil magnetic field with a dipole (or quadrupole) geometry and a surface induction $B_{s} \leq 10^{3} \mathrm{G}$, typically $B_{s} \approx 100-200 \mathrm{G}$. Such a field is detected in the slowly rotating star $\beta$ Cephei by Henrichs et al (1999). The disk may have a magnetic field of $\approx 10-20 \mathrm{G}$. To search for a magnetic field in fast rotating Be stars with disks it is useful to detect Zeeman broadening in spectral lines, polarization in the continuous spectrum and gyrosynchrotron radioemission.

Be stars with fossil magnetic fields and magnetic accretion disks should have magnetospheres with radius $r \approx R_{d}=R_{m} \approx 3 R_{\star}$, which is comparable to the radius of the pseudophotosphere (see Harmanec, this volume). In this region the value of the magnetic field of the disk is approximately the same as the value of the stellar dipole magnetic field. Under such circumstances a current sheet should form at the nagnetospheric boundary. Reconnection of oppositely directed magnetic fields of the disk and the star can produce X-ray emission.

\section{References}

Cowling, T.G. 1945, MNRAS 105, 166

Dudorov, A.E. 1990, in Itogy Nauki i Texniki, VINITI, Iss. 39, 77 (In Russian)

Dudorov, A.E. 1995, Astron. Reports 39, 790

Dudorov, A.E. 1998, Izvestia Akademii Nauk, Seria Fisicheskaya 62, 1759

Dudorov, A.E., Tutukov, A.V. 1990, Sov. Astron. 34, 171

Dudorov, A.E., Zhilkin, A.G., Kuznetsov O.A. 1999a in Numerical Astrophysics, Kluwer Ac. Publ., 1999, 240, 389

Dudorov A.E., Zhilkin A.G., Kuznetsov O.A. 1999b Mat. Modelirovanie 101, 101 (In Russian)

Henrichs, H.F. 2000, this volume

Mestel, L. 1967, in The Magnetic and Related stars, Cameron, A.G.W., Ed., Maltimore: Mono Book, 1967, 101

Shakura, N.I., Sunyaev 1973, A\&A 24, 337

Spitzer, L. 1958, in Electromagnetic Phenomena in Cosmical Physics (IAU Symp. no.6) Lehnert, B., Ed. Cambridge: Cambridge Univ., 1958, 169 